ORIGINAL

PROF-1088

\title{
SURGERY FOR LOWER LID SENILE ECTROPION;
}

\section{LATERAL TARSAL STRIP VERSUS FULL THICKNESS PENTAGONAL RESECTION}

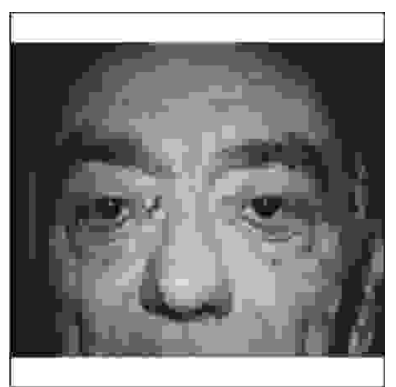

\author{
DR. RAO MOHAMMAD RASHAD QAMAR \\ MBBS, MCPS, FCPS \\ Assistant Professor of Ophthalmology, \\ B.V.H / Q.A.M.C, Bahawalpur, Pakistan \\ DR. MUHAMMAD A AHAD \\ Kent County Ophthalmic Hospital, \\ Maidstone UK \\ DR. CHUNG N CHUA \\ Kent County Ophthalmic Hospital, \\ Maidstone UK
}
Dr. Carole A Jones
Kent County Ophthalmic Hospital, Maidstone UK

Dr. Tariq Mahmood Araian

Associate Professor

QAMC Bahawalpur

\begin{abstract}
Objectives: To compare the results of lateral tarsal strip procedure with full thickness pentagonal lid resection in the correction of involutional ectropion. Material and Method: A retrospective study of 102 patients who underwent surgery for senile ectropion of lower lid. All the patients underwent either lateral tarsal strip operation or full thickness pentagonal resection. On the basis of signs and site of ectropion, the patients were divided into three main groups; 1) Medial ectropion, 2) General ectropion and 3) ectropion with chronic lid margin changes. Success was defined as relief of symptoms and good lid position. Results: $69 \%$ of patients had lateral tarsal strip and $31 \%$ of patients had full thickness pentagon resection. Overall success was achieved in $90 \%$ patients who underwent lateral strip operation and in $91 \%$ patients with full thickness pentagonal lid resection. In patients with medial ectropion the success rate was $87 \%$ in lateral tarsal strip procedure compared to $96 \%$ with full thickness lid resection. In general ectropion group, the success rate was $92.5 \%$ with lateral tarsal strip and $80 \%$ with full thickness lid resection. In patients with secondary lid margin changes the success rate was $74 \%$ in lateral tarsal strip procedure compared to $87 \%$ with full thickness pentagon resection. Conclusion: Both lateral tarsal strip and pentagon resection are effective in the treatment of senile lower lid ectropion,
\end{abstract}

Key words: Ectropion, eyelid, involutional

\section{INTRODUCTION}

Senile ectropion of the lower lid is a common cause of eyelid mal-position encountered by the ophthalmologists. It is associated with structural eyelid changes that 
include disinsertion of the lower lid retractors, laxity of medial and lateral canthal tendons and atrophy and degeneration of the orbicularis muscle ${ }^{1}$. The lateral canthal tendon is more prone to laxity than the medial canthal tendon. The tarsal section of the lower eyelid being stronger tends to stretch less than the tendons ${ }^{2}$. All these changes lead to stretching or elongation of lower lid leading to a point of ectropion formation. Epiphora occurs as the punctum moves away from the globe and this is exacerbated by the concurrent eyelid laxity. In chronic ectropion punctal stenosis, conjunctival keratinisation \& hypertrophy, tarsal thickening and skin contracture develops leading to ocular irritation. The goal of surgical repair is to re-establish the anatomical \& physiological relationship of the lacrimal punctum and lower eyelid margin to the globe.

Two popular procedures for correcting involutional ectropion of the lower lid are lateral tarsal strip (LTS) procedure and full-thickness lid resection (FTLR). There has no been study comparing these two procedures although $\mathrm{Liu}^{3}$, had compared various techniques of lid shortening undertaken for a range of lid mal-positions. This study compares the outcomes of lateral tarsal strip procedure with full thickness pentagon lid resection in senile lower lid ectropion.

\section{MATERIAL AND METHODS}

We reviewed retrospectively all patients who underwent surgery for lower lid senile ectropion from March 1999 to October 2005. All the operations were performed or supervised by one surgeon. The surgery performed was either lateral tarsal strip operation or full thickness pentagon resection. Excluded from the study were patients with significant medial canthal tendon laxity (where the puntum can be pulled laterally beyond the medial limbus with the eye in primary position), lacrimal duct obstruction and previous lid surgery. All patients had full lower lid evaluation preoperatively including measurement of horizontal lid laxity, medial and lateral canthal tendon laxity, patency of the lacrimal system, location of ectropion (either medial or generalized) and any secondary lid margin changes. According to the site and signs of ectropion, patients were divided into following groups;

1) Medial ectropion

2) General ectropion

3) Ectropion with chronic lid margin changes and

4) Ectropion with no lid margin changes.

All Patients with medial ectropion had medial diamond tarso-conjunctival excision in addition to lid shortening procedure.

The patients were reviewed on the 10th postoperative day and then 6 weeks postoperative. At each visit the position of lid margin was assessed and patients were enquired regarding the degree of symptomatic relief. Success was defined as relief of symptoms and good lid position. The success rates for the two procedures were compared for all as well as different types of involutional ectropion.

\section{RESULTS}

102 eyes of 78 patients fulfilled the criterion for the study. There were 48 females and 30 males. The age ranged from 61 to 96 years (mean of 76 years). 70 eyes underwent lateral tarsal strip and 32 full thickness lid resection. The follow up ranged from 6 weeks to 78 months (mean of 14.4 months).

Horizontal lid laxity \& Lateral canthal tendon laxity was present in all 102 cases. Mild medial canthal tendon laxity (not necessitating medial canthal surgery) was present in 11 out of 102 cases. 38 eyes had chronic lid margin changes i.e. keratinisation of the tarsal conjunctiva due to long-standing ectropion. 52 patients had medial ectropion and 50 patients had general ectropion. All the cases with medial ectropion had medial tarsoconjunctival diamond excision along with lid shortening procedure.

The demographic details and baseline characters are shown in (Table I) and the types of ectropion in the both surgical groups are shown in (Table II).

All patients with medial ectropion also underwent medial 
tarso-conjunctival excision. Of the 70 patients in the LTS group, 30 also had concurrent medial tarso-conjunctival excision. Of the 32 patients in the FTLR group, 22 also had concurrent medial tarso-conjunctival excision.

\begin{tabular}{|c|c|c|}
\hline \multicolumn{2}{|c|}{ Table-I. Basis line characteristics of the two surgical groups } \\
\hline & $\begin{array}{c}\text { Lateral Tarsal Strip } \\
(\mathbf{7 0})\end{array}$ & $\begin{array}{c}\text { Full Thickness Lid } \\
\text { Resection (32) }\end{array}$ \\
\hline Age & $61-92(75.65 \pm 6.8)$ & $62-96(76.34 \pm 8.8)$ \\
\hline Male : Female & $1: 1.6$ & $1: 1.5$ \\
\hline $\begin{array}{c}\text { Duration of } \\
\text { Ectropion }\end{array}$ & $\begin{array}{c}6-36 \text { Mean } \\
(16.2 \pm 7.2)\end{array}$ & $6-36(17.34 \pm 7.98)$ \\
\hline $\begin{array}{c}\text { Follow up } \\
\text { (Months) }\end{array}$ & $1.5-56(15.0 \pm 13.1)$ & $2-78(18.44 \pm 16.6)$ \\
\hline
\end{tabular}

\begin{tabular}{|c|c|c|c|}
\hline \multicolumn{4}{|c|}{ Table-II. Types of Ectropion and Surgery } \\
\hline $\begin{array}{c}\text { Types of } \\
\text { Ectropion }\end{array}$ & $\begin{array}{c}\text { Lateral Tarsal } \\
\text { Strip }\end{array}$ & $\begin{array}{c}\text { Full Thickness } \\
\text { Lid Resection }\end{array}$ & Total \\
\hline Medial Ectropion & 30 & 32 & 102 \\
\hline General Ectropion & 40 & 22 & 52 \\
\hline $\begin{array}{c}\text { Secondary lid } \\
\text { Margin Changes }\end{array}$ & 23 & 10 & 50 \\
\hline
\end{tabular}

The surgical outcomes are shown in (Table III). The overall success rates were comparable between the two groups, with each group achieving more than 90\% success rate. In patients with medial ectropion the FTLR $(95.5 \%)$ showed better results as compared to LTS (86.6\%) but the difference was not statistically significant ( $p>0.05)$. In patients with general ectropion, LTS (92.2\%) had better success rate than FTLR (80\%) but again the difference was not statistically significant $(p>0.05)$. In patients having long-standing ectropion, the FTLR $(86.6 \%)$ achieved a better results compared with LTS $(73.9 \%)$.

This difference is again not statistically significant $(p=0.88)$. But comparing the success rate between patients with long standing ectropion (patients with lid margin changes $n=38$ ) and patients with fairly recent ectropion $(n=64)$ the lids with recent onset ectropion fared better. The success rate was $96.8 \%(62 / 64)$ in patients with fairly recent ectropion compared to $78.9 \%$ $(30 / 38)$ in patients with chronic lid margin changes. $(p=$ $0.01)$.

\begin{tabular}{|c|c|c|c|}
\hline \multicolumn{5}{|c|}{ Table-III. Success rate of both types of surgeries } \\
\hline & Lateral Tarsal Strip (70) & Full Thickness Lid Resection (32) & Total (102) \\
\hline Over all (102) & $90 \%(63 / 70)$ & $90.6 \%(29 / 32)$ & $90.2 \%(92 / 102)$ \\
\hline Medial ectropion (52) & $86.6 \%(26 / 63)$ & $95.5 \%(21 / 22)$ & $90.4 \%(47 / 52)$ \\
\hline Gen. Ectropion (50) & $92.5 \%(37 / 40)$ & $80 \%(8 / 10)$ & $90.0 \%(45 / 50)$ \\
\hline $\begin{array}{c}\text { Ectropion with lid changes (38) } \\
\begin{array}{c}\text { Ectropion with no lid changes } \\
(64)) 97.8 \%(46 / 47) 94.1 \% \\
(16 / 17) 96.8 \%(62 / 64)\end{array}\end{array}$ & $73.9 \%(17 / 23)$ & $86.6 \%(13 / 15)$ & $78.9 \%(30 / 38)$ \\
\hline
\end{tabular}

\section{COMMENTS}

The aim of ectropion surgery is to re-oppose the lid to the globe and reduces epiphora. Horizontal lid shortening involving the tarsus plate has traditionally been the treatment of choice. Several procedures have been described dating back to Adam's full thickness triangular eyelid resection in 1812. Smithpopularized the technique of full-thickness resection of the eyelid, and this was the preferred procedure for ectropion repairs for many years. In 1977 Tenzel $^{4}$ first described the lateral 
canthal sling for senile ectropion, focusing attention on tightening the slack lateral tendon. This was modified by Anderson ${ }^{5}$ into the lateral tarsal strip procedure which is gaining popularity. While the technique addresses the problem of lateral canthal tendon laxity seen in many involutional ectropion, it is difficult to master since the periostium is not visualized directly when anchoring the tarsal strip to the orbital periostium giving rise to canthal dystopia. ${ }^{6}$ This occurs when the suture is placed too low and anteriorly in the periostium of lateral orbital wall causing post operative lid retraction.

Temporal tenderness and suture granuloma are other complications. In this study 10 patients with lateral tarsal strip developed temporary tenderness at the suture sites that resolved in 1-8 weeks time. With regard to the eyelid, the main complication is persistent punctal ectropion. In the LTS group, we had six eyes with persistent punctual ectropion. Preoperatively, five of these six eyes had medial ectropion and a tarsoconjunctival incision was performed. Of the three recurrent ectropion in the LTS group, two underwent FTLR and one further LTS.

Full thickness pentagonal resection is also a wellaccepted lid tightening procedure. When properly executed the results are excellent and there is minimal scarring. In case of medial ectropion, concurrent diamond tarso-conjunctival excision as lazy- $\mathrm{T}^{7}$ gives good surgical results. Placement of intratarsal and lid margin sutures is relatively time consuming, but the procedure is more easily undertaken by a general ophthalmologist. Complications include lateral displacement of the punctum especially in the presence of lax medial canthal tendon, phimosis of the palpebral aperture, lid notching and trichisis. In this study, one eye with FTLR developed lid notching and one eye developed trichisis. The eye, which developed trichisis, had a long-standing ectropion with secondary lid margin changes. One eye with FTLR developed recurrence which was corrected with LTS.

The overall results for both procedures were similar and comparable with other studies ${ }^{3,8}$.
Although there was no statistically significant differences in the overall results, ectropions with secondary lid margin changes had a better surgical outcome when FTLR was performed compared to LTS. Similarly, in predominantly medial ectropions, LTS was not as good as FTLR (lazy-T $)^{7}$ procedure and this finding is consistent with other studies. However there was statistically significant relation between the success rate and the presence of secondary lid changes. Overall $96.8 \%(62 / 64)$ of patients who had no chronic lid changes (including both procedures) had successful surgery as compared to $78.9 \%$ (30/38) with secondary lid margin changes $(p=0.02)$. If we compare procedures between the two groups, we see that there is no significant difference in the success rate if the FTLR was performed for lids with secondary lid margin changes or not; but in case of LTS, the success rate was $97.8 \%$ (46.47) in lids with no margin changes, which dropped to $73.9 \%(17 / 23)$ in patients with lid margin changes $(p=0.01)$. (Table III). This may be explained by the fact that the thickened and inflamed conjunctiva and eyelids were not resected in the LTS as is the case in FTLR. The presence of these lid changes in the LTS can continue to irritate the eye and causes epiphora thus symptoms of watering and irritation persist.

The main weakness in this study was that the patients were not randomly allocated to the two surgical techniques. Therefore, the types of ectropions in both groups were not equally distributed. This may explain why the results did not reach statistical significance.

In summary, our study shows that both LTS and FTLR are effective for all types of involutional lower lid ectropions but the success rate differ for different types of ectropion. LTS is preferable to FTLR in patients without predominantly medial ectropion or chronic eyelid changes. Eyelids with chronic changes and predominantly medial ectropion have a higher success rate with FTLR (including lazy T) than with LTS (including concurrent tarso-conjunctival excision). Thus whilst shortening is the aim of ectropion surgery the type of procedure should be tailored to the site of the ectropion and the duration of lid malposition. 


\section{REFERENCE}

1. Vallabhanath $P$, Carter SR, "ectropion and entropion" Current Opinion in Ophthalmology 2000; 11: 345-351.

2. Anderson RL, "tarsal Strip Procedure for Correction of Eyelid Laxity and Canthal Malposition in the Anophthalmic Socket" Ophthalmology 1981; 88: 895903.

3. Liu, D.T Lower eyelid tightening: a comparative study. Ophthalmic Plastic and Reconstructive Surgery 1997; 13 3 199-203.

4. Tenzel RR, Buffam FV, Miller GR: The use of the "lateral canthal sling" in ectropion repair. Can J Ophthalmol 1977; 12: 199.
5. Anderson RL, Gordy DD: The tarsal strip procedure. Arch Ophthalmol 1979; 97:2192.

6. Orcutt JC: Lateral Canthal Surgery. Arch. Facial Plast. Surg vol 2, Jan-March 2000.

7. Smith B: The Lazy-T correction of ectropion of the lower punctum. Arch. Ophthalmol. 1976; 94:1149-1150.

8. Baker SS: Lateral Canthal Tendon Suspension using Carbon dioxide Laser. Dermatol. Surg. 1995; 21: 10711073.

\section{URO-SPECIAL CARD}

Free consultation by medical officers

24 hours service throughout the year Emergency services $100 \%$ discount on medical officer fee on hospitalization

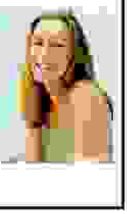

\section{G SHAFFE MEDICAL GeNTRE}

175. JINNAH COLONY, FAISALABAD

TEL: + $92412617122-24$, FAX: +92412623413

editor@fsd.paknet.com.pk 